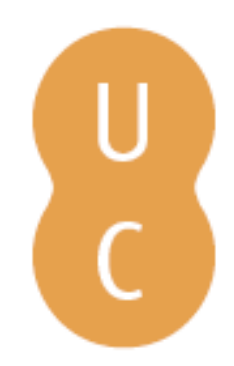

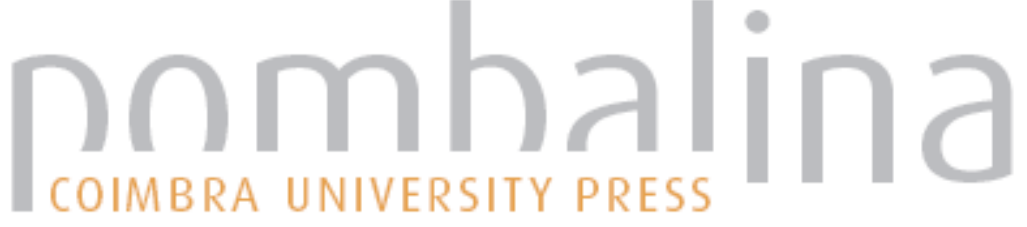

\section{Un patrimonio culturale "nascosto": il Parco Nazionale della Sila}

\author{
Autor(es): $\quad$ Faccioli, Marina
}

Publicado por: Imprensa da Universidade de Coimbra

URL

persistente:

URI:http://hdl.handle.net/10316.2/43439

DOI:

DOI:https://doi.org/10.14195/978-989-26-1475-5_10

Accessed : $\quad$ 26-Apr-2023 14:31:35

A navegação consulta e descarregamento dos títulos inseridos nas Bibliotecas Digitais UC Digitalis, UC Pombalina e UC Impactum, pressupõem a aceitação plena e sem reservas dos Termos e Condições de Uso destas Bibliotecas Digitais, disponíveis em https://digitalis.uc.pt/pt-pt/termos.

Conforme exposto nos referidos Termos e Condições de Uso, o descarregamento de títulos de acesso restrito requer uma licença válida de autorização devendo o utilizador aceder ao(s) documento(s) a partir de um endereço de IP da instituição detentora da supramencionada licença.

Ao utilizador é apenas permitido o descarregamento para uso pessoal, pelo que o emprego do(s) título(s) descarregado(s) para outro fim, designadamente comercial, carece de autorização do respetivo autor ou editor da obra.

Na medida em que todas as obras da UC Digitalis se encontram protegidas pelo Código do Direito de Autor e Direitos Conexos e demais legislação aplicável, toda a cópia, parcial ou total, deste documento, nos casos em que é legalmente admitida, deverá conter ou fazer-se acompanhar por este aviso.

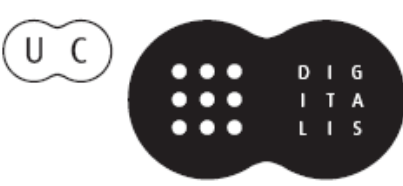




\section{UN PATRIMONIO CULTURALE “NASCOSTO”. IL PARCO NAZIONALE DELLA SILA}

DOI https://doi.org/10.14195/978-989-26-1475-5_10

MARINA FACCIOLI

LE ARTICOLAZIONI DELLA STORIA TERRITORIALE

Le tipologie presentate dalle configurazioni insediative locali come risultanti dei processi di aggregazione territoriale si sono ricondotte nella evoluzione dell'area silana alle storiche problematiche territoriali della regione calabrese e, per più versi, dell'intero Mezzogiorno.

Le condizioni ambientali e le dinamiche del lavoro e della cultura dei gruppi umani che in quelle terre hanno agito hanno progressivamente condotto alla definizione di individualità territoriali diversamente definite nel tempo, regioni o subregioni, i cui confini si sono tracciati nel quadro di situazioni umane, eventi politici spesso improntati da dinastie ecclesiastiche o baronali, processi economico-produttivi, dinamiche di integrazione socio-territoriale.

Da questo punto di vista possiamo guardare alla storia dell'Altopiano della Sila attraverso le metodologie dell'analisi toponomastica che, nel quadro di una grande, importante tradizione degli studi geografici ha studiato, alla luce dei nomi assegnati ai luoghi la natura della consapevolezza che gli abitanti si sono costruiti a vantaggio della propria comunità, sulla base delle proprie relazioni di appartenenza al medesimo luogo e, insieme, coerentemente con l'affermazione della propria individualità rispetto ad altri gruppi e altri luoghi, anche contigui.

Come già visto il nome odierno, assegnato alla Sila per caratterizzare una regione originariamente naturale, situata in un'area tipica della "penisola" calabrese coperta di boschi e foreste ha, insieme, definito una regione la cui 
specificità è consistita in una "vocazione" economica costruita sulla pratica dell'uso produttivo del legname, già nota a geografi e cartografi greci e romani. Dunque, una individualità originale, precisamente delimitata come formazione nell'ambito della morfologia orografica regionale, il cui tipico paesaggio è costituito da un altopiano lievemente ondulato, solcato al proprio interno da direttrici rilevate ricoperte di boschi e frequentato essenzialmente da attività del pascolo e della pastorizia.

Di interesse essenziale, tuttavia, per l'acquisizione del significato delle funzioni territoriali della regione silana, oggi, sono ripartizioni territoriali di più fine lettura che, se hanno in più casi trovato riscontro in puntuali divisioni giurisdizionali come i comuni, tuttavia sono leggibili a diverse scale nella storia e nell'interpretazione delle azioni dei gruppi umani, nel lavoro di conquista e di sfruttamento delle risorse del suolo e nelle dinamiche relazionali fra diverse società locali.

Dunque le tradizionali "regioni" del territorio silano si sono costituite come insiemi di paesaggi tracciati nella natura e, insieme, nella storia culturale del territorio, con significati strategico-politici, naturalistici, sociali. Le vicende culturali delle popolazioni hanno progressivamente disegnato $i$ confini di un ordine topografico-giurisdizionale in cui le aree comunali risultano come "esili e prolungate lingue di terra" (Gambi, 1965, p. 201) che risalgono, a ovest dalla valle del fiume Crati e a est dalla linea costiera ionica, lungo i fianchi dell'altopiano, secondo i tracciati disegnati dalle incisioni delle fiumare. Quelle partizioni amministrative esprimono la storia tradizionale di popolazioni che in quei luoghi hanno ripercorso diversi suoli e sperimentato vari assetti colturali, superando differenti limitazioni climatiche e vegetazionali, dalla collina a diverse altezze del bosco.

In età moderna le partizioni comunali hanno raccolto leredità di vicende antiche, segnando le coincidenze fra diversi ambienti e vicende dellorganizzazione impartita dalle società umane, a indicare la reciproca integrazione che le attività del lavoro producevano fra il mare e l'interno, in una continuità di intenti tradizionalmente rivolta a vincere le asperità dei terreni e la variabilità del clima, a sconfiggere la miseria, a produrre e incrementare coesione sociale.

A tale proposito le vicende dellaltopiano silano vennero fortemente condizionate nell'ambito delle vicende di istituzione dei "distretti" di Cosenza e Catanzaro, perché quelle vicende segnarono definitivamente il peso delle due città nel territorio regionale. Da un lato, infatti la Sila rappresenta- 
va il nucleo primario del distretto cosentino, a fianco della valle del Crati, importante risorsa per il mercato del vicino capoluogo, grazie all'elevata fertilità dei terreni agricoli; dallaltro, nei versanti orientali dei rilievi presilani si costituì un contrafforte non solo ambientale ma di grande portata socio-culturale, fra il Crotonese e il Catanzarese, dove, a partire dal $\mathrm{IX}^{\circ}$ secolo, con l'appoggio del monachesimo di provenienza orientale, andarono a raccogliersi le popolazioni dei paesi fondati dai Greci lungo le coste comprese fra i golfi di Sibari e Squillace.

Fra le età moderna e contemporanea le dinamiche di regionalizzazione locale sono state attivate sulla base di eventi e processi complessi, primi fra tutti quelli riconducibili alle grandi iniziative di riassetto del territorio agricolo, come le riforme fondiarie e i grandi lavori di bonifica idraulica. Queste dinamiche, dunque, che hanno interessato fondamentalmente le potenzialità e le risorse della storia sociale del Mezzogiorno, hanno impresso altri ritmi e hanno accordato altre scale di intervento ai grandi processi di trasformazione territoriale, definendo le linee della attuale configurazione regionale dell'area silana. Conviene, a tale proposito, rifarsi alle caratteristiche strutture fondiarie che si sono andate affermando nel nostro territorio, un ambiente a gestione estensiva e latifondistica, in parte organizzato da grandi aziende e dominato da rapporti tipicamente capitalistici, per l'altra parte impostato secondo complessi rapporti di origine tipicamente contadina, con imprese di minima entità, dal punto di vista sia dimensionale sia produttivo.

L'organizzazione socio-economica dell'altopiano deriva, a tutt'oggi, dallo smembramento di grandi aziende cerealicolo-pastorali in cui le tradizionali differenziazioni delle vicende insediative locali si erano, in più casi, manifestate attraverso diverse combinazioni delle consuetudini del lavoro contadino, fra le attività della pastorizia, con il pascolo degli ovini, e la coltura estensiva dei cereali, nel tentativo costante di sfruttare nel modo ottimale i vantaggi del mite clima invernale e di sfuggire all'aridità e al pericolo di infezioni nei mesi estivi. Manifestazioni tipiche si erano presentate attraverso i movimenti di transumanza e le periodiche, e tuttavia intense, relazioni fra popolazioni montane e costiere, fra gli abitanti dei villaggi interni situati alle maggiori latitudini e quelli insediati lungo le coste, dove i primi, in inverno, conducevano al pascolo il bestiame e, insieme, cercavano lavoro stagionale o aree in cui fosse possibile seminare. 
Dunque le forme di regionalizzazione agricola che hanno interessato l'altopiano silano caratterizzandone la moderna organizzazione hanno tratto impulso da circuiti culturali che legavano in stretta interazione le fasi di permanenza e di migrazione fra montagna e coste, fra cicli stagionali e difficili, ripetitive esperienze di lavoro, spesso esclusivamente finalizzate alla sussistenza.

I paesaggi diversi che oggi caratterizzano la Sila, conferendo fascino e originalità all'area del Parco, ripropongono usi del suolo e dinamiche insediative e organizzative impostate essenzialmente in seguito ai lavori di bonifica integrale condotti nella regione nel corso del $\mathrm{XX}^{\circ}$ secolo. La "rinascita" delle aree dell'altopiano seguì a due primarie tipologie di intervento agrario. Anzitutto, la sistemazione dei pendii franosi lungo i versanti montuosi e il riequilibrio del manto vegetazionale, operazioni essenziali per la sopravvivenza delle aree pianeggianti ai piedi dellaltopiano e, dunque, per la difesa dello stesso altopiano dall'isolamento a valle, oltre che per il riassetto delle condizioni degli stessi terreni posti a maggiori altitudini. Altra operazione basilare consistette nell'imbrigliatura dei corsi d'acqua al fine di controllare il trasporto di materiali verso le pianure e predisporre un piano di difesa contro le inondazioni.

Come più volte rilevato dagli studiosi del nostro Mezzogiorno (Gambi, 1965) ogni processo territoriale che ha consentito di individuare forme e tipologie di articolazione regionale e subregionale ha registrato nel Meridione dinamiche tipiche, contrassegnate dalla mancanza di interazione stabile fra soggetti e realtà organizzativi e produttivi. Anche i paesaggi silani si sono andati configurando nel passato secondo tratti che hanno conferito ai luoghi una indubbia originalità e, insieme, hanno generato condizioni penalizzanti, in assenza di una chiara distinzione fra le società della montagna e della collina e, conseguentemente, senza una vera autonomia funzionale e una consolidata identità delle condizioni culturali e produttive. Piuttosto, si è affermata una realtà tipica delle società pastorali, necessariamente integratasi con la frequentazione di ambienti diversi, in pianura, e dunque con condizioni climatiche, paesaggistiche, socio-culturali di altra tradizione e di diversa forza organizzativa. Le attività e le aggregazioni territoriali locali si sono presentate spesso associate perché hanno reso interattivo lo sforzo costante diretto a rendere fruibili i terreni, a superare con il lavoro gli ostacoli e i vincoli ambientali, mediante pratiche agrarie di integrazione fra montagne e pianure, soprattutto attraverso l'abitudine imposta dalla pratica stagionale del nomadismo, periodicamente riproposta dalla transumanza. 
Su tradizioni di questo tipo, in cui gli ambienti tradizionali della vita locale hanno richiesto un apprendimento costante e differenziato attraverso attività ancora largamente primitive, piuttosto che pratiche evolute e ampiamente condivise, soltanto le forme di vita affermatesi nelle fasi recenti e attuali hanno portato trasformazioni radicali e impresso nuove tipologie distintive anche alle forme dei paesaggi.

Il cambiamento è derivato dal diversificarsi della domanda di consumi, dalla ridistribuzione del reddito locale, dall'affermazione di nuove tipologie di uso dello spazio, dall'ampliamento e dal diversificarsi dei mercati, oltre che dal modificarsi della scala degli interventi legato al salto nell'uso delle tecnologie.

Dunque, nel contesto del rinnovamento dei processi e delle forme di assetto del territorio silano, come nei casi di tante altre regioni montane del Paese in età contemporanea, va rimarcato come l'evento più fortemente significativo sia stato rappresentato dallesplosione delle attività turistiche e dal rivoluzionarsi dei modelli insediativi, relazionali ed economici che a quella esplosione sono seguiti. In questo contesto il progetto stesso di istituzione del Parco Nazionale si è progressivamente andato a configurare come "necessaria" risultante delle tradizionali operazioni di intervento locale richieste dalle esigenze tipiche del territorio.

La conoscenza delle vicende ambientali tradizionali e dei modelli storici di convivenza sociale ha condotto, infatti, a identificare e definire gli stessi progetti di tutela e valorizzazione delle risorse dei luoghi come "necessari" fattori di organizzazione territoriale. Le iniziative strettamente specialistiche hanno infatti riguardato la ricostituzione del manto forestale e la riqualificazione di paesaggi originali, riproponendo in gioco quelle facoltà originali e competitive e quelle potenzialità produttive che, già in altre epoche, avevano consentito la sopravvivenza e, poi, diverse forme di sviluppo dei luoghi stessi.

Anche l'affermazione delle nuove attività connesse al turismo, tuttavia, ha trovato spazio in un contesto di evoluzione territoriale non solo di natura paesaggistica ma, più propriamente, strutturale. La moderna trasformazione di queste località è stata "decisa" dall'evoluzione del quadro funzionale complessivo, a sua volta legato soprattutto agli interventi, promossi dalla riforma agricola, di diffusione di una rete di irrigazione, di sistemi di coltivazione, di stabilizzazione delle attività della zootecnia e di potenziamento di attività artigianali e industriali tipiche. Questo insieme di fattori ha, 
infatti, innescato processi diversi di riqualificazione e valorizzazione delle risorse originali di luoghi e paesaggi, garantendo, dunque, a vantaggio di questi, idonee condizioni di competitività per un accesso stabile al mercato.

\section{LA GESTIONE DEGLI ASSETTI PRODUTTIVI}

La programmazione relativa allassetto funzionale del territorio compreso nel Parco si inquadra, necessariamente, nell'ambito di dinamiche storiche di interesse più vasto, nel contesto dei processi funzionali che hanno preso forma nell'intera regione calabrese. Essendo l'agricoltura e la selvicoltura, anche in riferimento all'agriturismo, risorse chiave per un moderno sviluppo del territorio del Parco, è particolarmente importante ripercorrere, sebbene qui soltanto per cenni, la storia della conduzione agraria locale: questo, con particolare riguardo all'età che è seguita alla riforma agraria dell'ultimo dopoguerra, al fine di inquadrare le vicende del territorio locale entro tradizionali, specifiche problematiche.

Fra le risorse locali che costituiscono input idonei all'avvio di processi di organizzazione funzionale del Parco in senso produttivo, come si vedrà più avanti, compaiono attività artigianali e manifatturiere, spesso di lontana tradizione. Contemporaneamente, è evidente come lo stesso risanamento dell'assetto agricolo in termini di razionalizzazione e modernizzazione si presenti, necessariamente, connesso a una ripresa e a un potenziamento in termini selettivi degli apparati del secondario e del terziario locali.

La storia degli assetti regionali delle strutture delle manifatture e dei servizi è utile anche alla lettura del ruolo dell'altopiano silano nel territorio.

Sussistevano produzioni di altissima qualità artigianale che diventarono prodotti dell'esportazione (come i merletti a tombolo o le coperte a disegni, pregiati lavori d'intaglio del legno e conserve alimentari), dunque a delineare un contesto socio-produttivo e territoriale già ricco di notevoli potenzialità competitive. Grande importanza acquisirono in quegli anni le industrie alimentari, fra cui soprattutto quella olearia, nonostante che la lavorazione, fatta eccezione per pochi oleifici, funzionasse ancora in forma primitiva.

La grave, complessiva disorganizzazione che ostacolava le opportunità di coesione e integrazione regionale, le condizioni di degrado delle aziende, la dispersione delle risorse e, soprattutto, il bagaglio storico di un'identità 
spezzettata in numerosi e deboli distretti hanno fatto in modo che difficilmente le iniziative intraprese a partire dalla metà del secolo scorso siano riuscite a imprimersi positivamente sul territorio. L'industria calabrese ha tuttora bisogno di condizione di autonomia e di apertura all'integrazione con contesti esterni, mediante una rete di servizi, l'incremento e la formazione dell'occupazione e una produzione più specializzata in grado di stimolare nuova imprenditorialità.

Dunque la Sila è stata negli anni Sessanta al centro di una importante fase dell'industrializzazione della Calabria, proprio per l'incidenza delle componenti ambientali che la contraddistinguevano e che hanno funzionato come fattori di localizzazione dell'industria; un ruolo di assoluta centralità è stato assunto dalla produzione forestale, oltre che dalle risorse idriche utili alla produzione d'energia idroelettrica. Proprio all'industria idroelettrica deve esser dato il merito di aver contribuito ad assegnare, nei primi 60 anni del Novecento, un valore nuovo e moderno all'area silana. L'elevazione e la grandezza dei suoi altopiani, circondati da versanti ripidi, la presenza di fiumi che rendono il suolo poco permeabile, hanno progressivamente individuato nella Sila una ottimale localizzazione per bacini artificiali.

In Sila, già dal XIX secolo, erano state aperte le due principali strade di transito: una, di forte rilevanza commerciale, utilizzata dagli anni Sessanta a scopo turistico, che da Cosenza arriva fino ai pascoli silani e, superato San Giovanni in Fiore, attraverso la Valle del Neto, raggiunge Crotone; laltra che, con i rami di Rogliano e Colosimi, porta dal bacino del Savuto sullaltopiano, per poi diramarsi in varie strade che smaltivano le produzioni forestali e congiungevano i paesi limitrofi.

Nelle modalità evolutive dell'organizzazione del territorio dell'altopiano silano entravano, intanto, negli anni della ripresa postbellica, nel periodo seguente alla seconda guerra mondiale, anche le nuove dinamiche tipiche del turismo, che si integrò fortemente con il complesso delle funzioni socio-produttive coeve, sotto l'aspetto della domanda di servizi moderni, di vie di transito e comunicazione e mediante lo sviluppo di nuove funzioni e morfologie territoriali di carattere urbano. Fu, anzi, proprio il turismo a segnare le tappe del passaggio a nuove, spesso innovative, forme di fruizione del territorio.

Fino agli anni Sessanta, infatti, viaggiare e visitare i frequenti villaggi nascosti fra i monti bruzi comportava incontrare condizioni di miseria (la Calabria era una delle regioni dove i consumi degli alimenti di base era 
bassissimo), in connessione, e in contraddizione, con paesaggi bellissimi che stavano avviando un turismo di transito che solo in piccola parte utilizzava le migliori linee viarie, complete di attrezzatura turistica. Distanti dagli itinerari più battuti, si andavano formando quelli che oggi sono i più attivi centri del turismo locale, allora villaggi montani, come Camigliatello Silano, Lorica, Mancuso. Ciascuno di questi villaggi, posti nel cuore della Sila, poteva allora ospitare circa 2000 persone che vi soggiornavano per diversi giorni e, per lo più, nei mesi estivi. Era, comunque, un tipo di turismo elitario, in tempi in cui questo tipo di vacanza era riservato alle più elevate classi locali: la Sila è stata, infatti, per lungo tempo meta dei cosentini e catanzaresi più facoltosi. Tuttavia, il tipo di turismo che più di altri riscuoteva successo era quello religioso, spesso legato a tipiche processioni e feste locali, in cui si celebravano produzioni originali e tradizioni del lavoro, in luoghi di culto in cui si ricordavano antichi riti stagionali.

Oggi, nel territorio del Parco le prospettive di progresso dell'economia, come si vedrà più avanti, sono fondamentalmente legate alla gestione delle risorse naturalistiche, mentre un grosso impulso potrebbe derivare proprio da un idoneo lavoro di rinnovamento e potenziamento del settore agrituristico. In forme significative, infatti, è tuttora presente l'artigianato, sparso un po' in tutti i comuni montani limitrofi alle aree del Parco: attività, queste ultime, in grado di incentivare non solo la produttività economica dei territori, ma anche la riappropriazione di valori e tradizioni socio-culturali. è importante sottolineare come la rivalorizzazione di processi e pratiche tipiche della vita di questi luoghi possa costituire una base per l'individuazione di nuove occasioni e modalità di sviluppo del turismo e, insieme, di condizioni di integrazione e coesione sociale fra diversi gruppi di attori locali.

\section{UN SISTEMA LOCALE?}

Lorganizzazione attuale del Parco si fonda, necessariamente, su condizioni di integrazione territoriale di carattere sistemico: infatti, perché unarea destinata a parco funzioni è essenziale che un insieme di fattori competitivi originali (risorse ambientali, sistemi produttivi, ambienti socio-culturali tipici, spesso a base familiare) operino, a loro volta, come elementi aggreganti di altri, diversi subsistemi del territorio in cui l'area stessa si inscrive. 
Lorganizzazione funzionale di un territorio, dunque, consente di leggere quel territorio in quanto "area parco". Il parco può essere tale in quanto funzionante come "ridisegno" della natura tipica locale (Donin, 1999); in quanto area propositiva di certe componenti aggreganti, a scala locale o "sovraterritoriale"; in quanto riproposizione di tradizioni insediative e di una urbanizzazione tipica; in quanto entità sistemica in cui trovano spazio e significato processi, sistemi e aree produttive a forte caratterizzazione. Lintegrazione che l'entità parco consente è effetto e funzione del riassetto costante e della capacità di autoconservazione del parco stesso.

In questi termini il Parco Nazionale della Sila si propone nei motivi dettati dalle diverse forme aggreganti che vi trovano spazio e che riorganizzano, a più scale, il territorio come unità sistemica. Dunque i contenuti originali funzionano come risorse competitive e patrimoni in grado di porre il contesto locale in connessione con una scala di relazioni più ampie, interregionali e internazionali, mediante la promozione di fattori di produzione e riproduzione di processi socio-economici e conoscitivi. Le attività originali dei luoghi che vivono nel Parco diventano fattori di affermazione e confronto nel territorio: l'istituzione del Parco rende, infatti, quei fattori competitivi e ne valorizza le potenzialità relazionali avviando azioni conoscitive specialistiche, mediante la guida lungo itinerari tipici e, dunque, favorendo la promozione dell'apprendimento di contenuti e dinamiche.

Tutto questo deve, tuttavia, necessariamente, avvenire entro le condizioni poste dalle normative che regolano gli interventi di gestione del territorio, con particolare riguardo al sistema di vincoli su cui le relative politiche si ordinano. Le funzioni previste nel progetto dellarea parco devono, dunque, concorrere a organizzare le linee di una specifica "sostenibilità" locale, entrando a far parte integrante delle dinamiche di coesione funzionale e sociale nell'ambito del territorio. Le attività previste dovrebbero, dunque, promuovere l'integrazione fra substrati tradizionali e interventi nuovi, mediante una sperimentazione continua di pratiche e processi produttivi e socio-culturali di "riaggiustamento" di mansioni e competenze aziendali, nell'ambito di attività rivolte alla salvaguardia dei paesaggi, nello svolgimento di funzioni ricreative, nel consolidamento e nella valorizzazione di pratiche artigianali e gastronomiche.

Il Parco silano funziona come luogo privilegiato di sperimentazione, come laboratorio specialistico per la ricostituzione delle componenti tipiche del milieu locale. Le produzioni originali riguardano funzioni antiche, di cui 
già si è ripercorsa la storia territoriale, fra cui la lavorazione del legno, una serie diversificata di lavorazioni artigianali e alcune colture tipiche, come quella dell'olivo. Nel quadro dei processi specialistici che qualificano il Parco come milieu originale entra anche la funzione turistica, che assume forme e modalità contestuali estremamente propositive, sebbene ancora in embrione, nell'agriturismo.

Le produzioni legate alla risorsa rappresentata dal legname si inquadrano in complesse dinamiche di gestione delle aree forestali in termini di "sostenibilità", in particolare nei termini delle relazioni fra operatori che gestiscono una materia prima di elevato pregio e domanda avanzata dal mercato.

E' importante ricordare come già la massiccia diffusione delle foreste, con particolare riguardo all'abbondante presenza di pinete (pino laricio) sia riconducibile a dinamiche di difficile gestione, nel tempo, dei suoli dellaltopiano silano. Infatti il pino, facilmente adattabile alla situazione ambientale locale, ha proliferato in accordo con condizioni di estremo degrado dei versanti e con l'erosione dei suoli dovuta alla deforestazione che nel tempo si è ripetuta nell'altopiano per conquistare pascoli e terreni per l'agricoltura itinerante.

La rilevanza che le pinete assumono nel territorio del Parco evidenzia l'importanza delle modalità di intervento funzionali alla conservazione dei relativi ecosistemi. La gestione forestale assume, tuttavia, un significato molto ampio perché non prende in considerazione solo l'aspetto produttivistico ma si inserisce in un quadro di salvaguardia di valori culturali, di pratiche di vita e di lavoro, in sostanza di riqualificazione di "saperi" locali, a loro volta connessi alla storia di tipologie proprietarie e di vicende economiche e sociali stratificatesi nel tempo.

Tipiche attività artigianali, espressione della cultura e della storia del lavoro in Sila sono quelle dell'intreccio e dell'intaglio, che riconducono proprio alle tradizionali lavorazioni di trasformazione delle risorse forestali. In questo settore si creano oggetti di varie forme, di uso quotidiano (sedie, cesti, ventagli, fusi) e autentici pezzi da collezione, di produzione limitata, quali i famosi bastoni, utensili domestici dalle tipiche forme locali, figure di animali spesso dal significato misterioso, legato ad autentiche leggende, attrezzi di lavoro che sono veri e propri cimeli culturali e spesso assumono anche significato scaramantico, a difesa e salvaguardia dei luoghi di vita e di lavoro familiare. 
L'istituzione del Parco come area di rispetto delle "qualitä" locali comporta, come già visto, precise valenze progettuali, fra cui trovano ampio spazio iniziative di rivalorizzazione di antiche attività artigianali sviluppatesi nell'altopiano silano, come la tessitura, il ricamo, la produzione di oggetti di ceramica e l'oreficeria. E' importante considerare come tali attività, essendo ormai in grado di confrontarsi con l'esportazione, consentano all'area silana livelli di competizione di interesse extralocale e internazionale, mediante relazioni strutturate nell'ambito di cooperative artigiane che oggi sostituiscono le antiche botteghe realizzando nuove, più razionali e operative, modalità di produzione e favorendo la creazione di occupazione in un quadro di rinnovata coesione sociale.

La tessitura ha avuto il suo centro a Longobucco, centro della Sila Greca situato lungo la valle, stretta e profonda, incisa dal fiume Trionto, già indicato dalla toponomastica locale come "anfratto" nascosto fra le rocce, in un contesto impervio e poco accessibile, isolato per gran parte dell'anno. Qui le donne divennero, nel tempo, tanto esperte nella ripetizione della pratica della tessitura di materie prime come la seta cruda, i cascami e la lana, da riuscire, via via, a perfezionarsi in attività altamente specialistiche e in produzioni di pregio elevato. Le lavorazioni avevano avuto origine dal riciclaggio di materiali poveri e di scarto, secondo una pratica sperimentata da numerosi centri di lavorazione tessile, affermatisi progressivamente come importanti distretti di produzione, in diverse aree del Paese. Tale attività, che ha qualificato in questo settore del territorio silano un vero processo produttivo "di nicchia", come manifattura altamente specialistica, con autentica funzione "interstiziale" fra produzioni più note e ampiamente diffuse, ha generato nel tempo l'acquisizione di professionalità competitive che, originatesi in contesti familiari, hanno acquisito un sempre più consolidato ruolo nel mercato.

Il ricamo, la produzione di tappeti secondo antiche tecniche greco-bizantine, arabe e persino egizie, disparate lavorazioni del cotone e del lino e una raffinata arte orafa si sono affermate a San Giovanni in Fiore, nel cuore dell'altopiano, fra Sila Grande e Sila Piccola, appena fuori dei confini del Parco, rappresentando preziose lavorazioni che costituiscono con le loro radicate prerogative culturali e socio-territoriali, "esternalità" preziose nei confronti dello sviluppo e della valorizzazione del Parco stesso. 
Nel corso della gestione del Parco silano sono emersi alcuni originali percorsi di crescita funzionale che propongono itinerari "specialistici" di sviluppo rivolti a qualificare l'area del Parco silano come sistema organico e integrato. Si tratta di attività con vocazione di "interfaccia" fra conservazione di patrimoni locali e crescita globale, per la propria stessa natura coerenti con prospettive di integrazione con le politiche di salvaguardia e sviluppo del Parco.

Fra queste è l'attività dell'agriturismo, pur ancora agli inizi, che dimostra potenzialità specifiche per la propria "appartenenza" territoriale alla natura tipica dei luoghi, fortemente individualizzati proprio nelle loro caratteristiche ambientali, climatiche e produttive. Questo itinerario progettuale, data la complessità e la diversità dei contenuti socio-ambientali che nel contesto del Parco prendono forma, pone in evidenza l'esigenza di una valutazione di patrimoni e risorse in un contesto più ampio dei confini assegnati al Parco. Quest'ultimo va inteso, piuttosto, come un'individualità territoriale composita e complessa, un sistema territoriale aperto e articolato entro limiti variabili, funzionali all'acquisizione e alla promozione delle proprie, diverse potenzialità di crescita culturale.

Una produzione che nellagriturismo troverebbe utilmente spazio per una riconversione qualitativa è quella legata allolivicoltura, di lontana tradizione, anzitutto perché soggetta a modalità di evoluzione strutturali nell'ambito della realizzazione locale di unagricoltura biologica di qualità. A questo proposito sono state condotte analisi mediante l'impiego di indicatori strutturali, organizzativi e strettamente produttivi, idonei a fornire informazioni sulle condizioni di mercato e sugli assetti decisionali e gestionali con cui confrontarsi: anche in questo caso la contestualizzazione dei confini territoriali del Parco entro spazi più ampi e complessi di quelli formalizzati ha contribuito come fattore conoscitivo essenziale alla definizione dell'area come soggetto coerente con il proprio territorio.

Turismo COME FATtore DEL “CAMbiamento"

Il processo di valorizzazione delle risorse turistiche dell'altopiano silano ha preso forme definite, sebbene ancora limitate, in particolare nell'ultimo 
dopoguerra, come già visto, nel quadro del potenziamento di centri con funzione "nodale", direttrici di transito e relazioni commerciali rivolti a far uscire la regione da antiche condizioni di isolamento e marginalità.

Il turismo che è possibile praticare nel Parco fa riferimento a località sparse fra montagne, laghi, torrenti, boschi e sentieri che ripercorrono antichi itinerari culturali, nei luoghi più significativi della storia locale.

Il più importante fra i centri turistici del Parco è Camigliatello Silano, a 1278 metri di altitudine, nel comune di Spezzano della Sila, alla confluenza di diverse direttrici che consentono l'accesso all'altopiano. Situato ai piedi del Monte Curcio, fra immensi boschi di pino, abeti, faggi e castagni, è il centro propulsore dell'intera area, stazione di turismo invernale di notorietà internazionale, sede della Caserma del Corpo Forestale dello Stato fin dal 1913. Località dotata di notevole ricettività alberghiera, locali tipici attrezzature per lo sport e il tempo libero, è dotata di alcuni importanti edifici, fra cui Torre Federici, in unarea in cui Federico II amava cacciare con il falco, sfruttando le correnti d'aria; e l'ex Hotel Itas, in località Camigliati, un bellissimo edifico recentemente ristrutturato cheospita la sede dell'Oldcalabria Parco Letterario Norman Douglas .

Lorica, località che vanta un toponimo strettamente legato ai tipici caratteri ambientali, sorge a 1315 metri di altitudine nel cuore dell'altopiano silano, fra i comuni di S. Giovanni in Fiore e Pedace, alle pendici del Monte Botte Donato, incastonata in una vallata che circonda il Lago Arvo, protetta da una "corazza", da cui il nome, costituita da montagne ricche di boschi e fauna selvatica. Con Silvana Mansio, villaggio immerso in suggestive e bellissime pinete, costituisce un complesso turistico molto frequentato per gli sport invernali, dotato di buone strutture ricettive oltre che di botteghe d'artigianato e di produzioni tipiche, con una struttura ben conservata ed equilibrata sotto l'aspetto dell'edilizia residenziale.

Nel versante cosentino dell'altopiano, Croce di Magara e Fallistro, a oltre 1340 metri di altitudine, nei comuni di Spezzano della Sila e Spezzano Piccolo, sono località turistiche sorte su villaggi poderali istituiti in seguito alla riforma agraria, di grande interesse ambientale per la prossimità alla riserva di Fallistro "I Giganti della Sila”. Punto di forza di questi centri è la localizzazione nell'area "forte" dell'offerta ricettiva locale, grazie anche ai buoni collegamenti con Camigliatello Silano mediante la statale 107 e la Superstrada Paola-Crotone. 
Ancora nel versante cosentino dell'altopiano, ai limiti occidentali del Parco, sorgono località turistiche di grande interesse, affacciati ad altitudini considerevoli sulle sponde sud-occidentali del Lago Cecita, dove la bellezza dei luoghi si unisce a una intensa cura delle risorse e dei patrimoni paesaggistici e culturali. Moccone, o Mucone, a 1306 metri, sul corso del fiume omonimo, è uno dei primi villaggi turistici dell'intero altopiano, già luogo di sosta per i primi visitatori e trasportatori di legname, è attualmente collegato con gli impianti sportivi di Monte Curcio e del complesso "La Pagliara". Monte Scuro, a 1633 metri, avvolto quasi perennemente da fitta nebbia, ospita un complesso di villeggiatura in un paesaggio di rara suggestione, avvolto in un silenzio surreale. Fago del Soldato, a 1450 metri, nel comune di Celico, si è organizzato spontaneamente come centro turistico per le bellezze naturalistiche e in quanto luogo di sosta obbligata dei trasportatori di legname e porta di accesso alla Sila: una suggestione particolare interessa lorigine dello stesso toponimo, legata a una leggenda che narra di uno scontro fra un brigante e un militare, quest'ultimo poi trovato morto sotto un albero di faggio.

A 1405 metri di altezza, Fossiata, compresa all'interno del Parco, è la più bella foresta della Sila Grande, con risorse floristiche e faunistiche perfettamente salvaguardate, dotata di aree per la sosta e il pic-nic, sulle rive di limpidi ruscelli, in paesaggi incontaminati di rara bellezza.

Anche il turismo culturale riguarda l'altopiano silano sotto i più diversi aspetti, dalla scoperta di percorsi naturalistici celebrati fin dagli autori classici, alla conoscenza di itinerari percorsi dal monachesimo brasiliano nel corso del Medioevo, alla visita delle abbazie, delle costruzioni storiche e dei palazzi che hanno segnato le tappe più importanti della storia di questi luoghi.

Sotto il nome di "Sentieri di Virgilio" sono raccolti i percorsi che conducono alla scoperta della antica Sila, la silva Sila, con le sue impenetrabili foreste, con i suoi sconfinati pascoli, con le sue magiche e suggestive atmosfere, celebrate, fra gli altri, da Virgilio e Dionigi di Alicarnasso. Il percorso inizia dal Monte Gariglione e segue sentieri che si inoltrano in bellissimi boschi di faggi e abeti, risalendo, poi, lungo la valle del fiume Tacina.

I "Sentieri dei Basiliani” sono percorsi distribuiti lungo quelle vie che univano, attraversando i boschi, le comunità monastiche insediate nella regione silana. Sono strade tracciate nella Sila Greca, a fianco di valloni stretti e incassati fra i monti, lungo i quali scorrono fragorosamente torrenti dalle acque impetuose. Il percorso inizia dalla piccola chiesa di S.Onofrio, verosimilmente un eremitaggio basiliano, per risalire il torrente Colognati. 
Un altro itinerario estremamente interessante e suggestivo riguarda la visita ai luoghi in cui visse l'abate Gioacchino: si visita la casa natale, la chiesa parrocchiale, la fontana Fietitta presso cui l'abate sostava, la vigna in cui avvenne la sua conversione. L'itinerario prevede una sosta a Luzzi, presso il Monastero della Sambucina, la più antica abbazia circestense del Meridione, dove Gioacchino si ritirò al ritorno dalla Terra Santa.

Si tocca anche il Monastero di Corazzo, nel comune di Carlopoli per poi raggiungere San Giovanni in Fiore, attraverso la Sila Piccola Catanzarese e cosentina, sostando presso il Romitorio affacciato sul Lago Arvo, presso Lorica. Nel centro di San Giovanni in Fiore si può visitare la celebre Abbazia Forense, che conserva le spoglie dell'abate; il Centro Internazionale di Studi Gioachimiti e la relativa biblioteca; e il Museo demologico.

Oggi, strutture e infrastrutture messe in atto nell'ambito del Parco Nazionale si presentano in grado di favorire la più ampia diffusione delle funzioni turistiche, favorendo non solo attività ricreative e del tempo libero, ma anche incentivando la conoscenza delle peculiarità locali e la formazione di operatori specialistici.

Dunque, nel quadro di una crescente conoscenza delle molteplici espressioni del territorio locale e al fine di favorire la fruizione delle risorse, sono state istituite alcune idonee strutture formative e ricreative e, in accordo con la normativa europea, 19 Siti di Interesse Comunitario (SIC).

\section{VERSO QUALE TERRITORIO?}

Dunque, vaste e multiformi sembrano essere le prospettive di sviluppo del Parco Nazionale della Sila, che all'ambiente suggestivo e composito nei colori e nelle espressioni aggiunge segni importanti di storia culturale e umana, tracce significative di un passato, a volte di marginalità, in altri casi di protagonismo, pur limitatamente ad alcuni settori produttivi, tuttavia sempre con forti tratti di individualità e originalità.

Lofferta che il territorio del Parco presenta richiede modalità complesse e fasi continue di ridefinizione e riassetto, secondo dinamiche proprie di ogni processo di produzione in termini sociali, territoriali, culturali. La domanda che proviene dalleconomia "reale" dei luoghi del Parco interessa una idonea "educazione" ambientale e naturalista per fruitori e operatori locali, 
oltre all'esigenza di costante riqualificazione di patrimoni preziosi, naturali e antropici, secondo la messa a punto di professionalità specialistiche nei campi della tutela dei beni paesaggistici, culturali, economici e strategici.

L'integrazione dei processi del territorio, nelle forme più attente alla tutela occupazionale e produttiva, si propone come soluzione privilegiata delle problematiche tipiche della crescita locale, riguardo all'esigenza di poli di integrazione territoriale per la promozione del turismo, per la riqualificazione dell'artigianato e delle attività manifatturiere, per la preparazione e la formazione della manodopera.

Il modello del sistema territoriale che, come già detto in più casi, si propone come soggetto capace di realizzare la conservazione e il consolidamento delle attività del Parco, propone un insieme organico di funzioni non limitate a quelle amministrative, entro confini operativi predeterminati e statici ma, piuttosto, è chiamato a inscriversi in una rete complessa di sistemi dinamici, a partire dalla scala locale e da quella regionale. Il territorio compreso nel Parco non può, infatti, limitarsi a rappresentare un'isola privilegiata le cui esigenze di salvaguardia e rispetto ne pregiudichino l'accessibilità e la fruibilità ma è, piuttosto, chiamato, più propriamente che in passato, a far parte integrante di entità relazionali più ampie e aperte, come espressione di potenzialità strategiche funzionali all'intero territorio in cui opera.

Il Parco costituisce, sostanzialmente, un sistema locale aperto a processi di interazione con il territorio: le località situate nei dintorni dell'area protetta e, tuttavia, interessanti perché in grado di costituire input di crescita e ampliamento delle funzioni espresse dal Parco, sono numerose e fortemente caratterizzate dalla presenza di risorse e patrimoni culturali tipici.

Sebbene, fino ad oggi, la fruizione turistica dell'area del Parco si sia basata, essenzialmente, sulle attività di turismo naturalistico e di didattica ambientale, particolarmente significativa si avvia a divenire la programmazione turistica che integrerà l'offerta naturalistica a quella culturale, dialogando proficuamente con il tessuto socio-territoriale in cui il Parco si inserisce.

Nel cuore della Sila, il paese più grande dell'altopiano è San Giovanni in Fiore, a 1050 metri di altitudine, sorto attorno al 1200 in funzione della storica Abbazia, a sua volta edificata, come già visto, dall'abate Gioacchino nella località chiamata Fiore, sul corso dei fiumi Neto e Arvo. Il centro, diventato comune nel 1530, è oggi in una fase di forte espansione dell'attività commerciale e culturale, dopo il superamento di un lungo periodo di contrazione demografica legata allemigrazione. Presenta ampie risorse 
storico-artistiche, fra cui l'Archicenobio Florense, del XIII secolo, in stile circestense, e la Chiesa Matrice, del 1600, dedicata a Santa Maria delle Grazie, oltre a tradizioni culturali legate all'artigianato orafo e tessile.

Un centro di grande interesse, dotato di una fisionomia territoriale originale costituitasi nel corso di complesse vicende storiche è Longobucco (789 m. s.l.m.), antico centro di estrazione dell'argento che i Sibariti e poi i Crotoniati e i Romani utilizzarono per la coniazione delle proprie monete. Il centro, importante miniera argentifera ancora fra i secoli XII e XVI, fu base del brigantaggio ottocentesco finché, nel corso del Novecento venne interessato da ampi lavori di rimboschimento e bonifica. Oggi, posto ai limiti settentrionali del Parco silano, attrae qualificati progetti di riorganizzazione territoriale funzionali all'espansione turistica e alla riqualificazione delle ricche produzioni locali. Fra i patrimoni artistici da visitare, la Chiesa Matrice, del XVIII secolo, dedicata all'Assunta, dal 1960 Santuario mariano; la Chiesa Santa Maria Maddalena, del secolo XVI, compresa nel complesso conventuale dei Riformati; e la chiesa di San Domenico, del XVI secolo, annessa al Convento dei Domenicani.

Sulla strada statale 282, nel versante nord-orientale della Sila Grande, a 870 metri s.l.m. sorge Bocchigliero, di antichissima origine, fondata da coloni romani dediti alla pastorizia, oggi una sorta di "paese-albergo" organizzato durante l'intero corso dell'anno come una grande e articolata struttura ricettiva. Le risorse artistiche, fra cui il Santuario di Santa Maria de Jesu, noto come Madonna delle Nevi, del 1700, e l'antichissima Torre del Campanile situata nel centro dellabitato, sono ampiamente diversificate, comprendendo anche ruderi di origine romana, resti di una necropoli bruzia e grotte neolitiche.

Ai margini nord-occidentali dell'altopiano silano sorge una serie di abitati che formano una fitta trama insediativa degradante verso la valle scavata dal Crati, a conferma di una intensa storia territoriale legata alla prossimità dell'area di espansione urbana di Cosenza.

Il centro più popoloso della zona presilana è Spezzano della Sila, a 780 metri di altitudine, attraversato dalla strada statale 107 e dalla Ferrovia della Calabria, fondato nel X secolo da profughi cosentini scampati alle invasioni dei Saraceni. Oltre ad altri bei monumenti sono da visitare il centro storico e l'imponente Convento che sovrasta l'abitato, costruzione ricca di opere d'arte, edificata da San Francesco di Paola, in onore del quale gli abitanti locali organizzano ogni anno, in settembre, una importante festa religiosa e civile. 
Ancora nel versante cosentino delle pendici silane, su di un terrazzo che sovrasta il capoluogo, si può visitare San Pietro in Guarano, a 640 m. s.l.m., costituito da un abitato che si estende dalla località di Fago del Soldato fino al corso del Crati. Dotato di importanti monumenti, fra cui la Chiesa Parrocchiale dedicata a Santa Maria in Gerusalemme (XIX secolo), costituisce una tappa sulla via di accesso al Parco silano soprattutto perché centro di villeggiatura estiva in cui si svolgono interessanti e originali manifestazioni di carattere folcloristico. E, sulla statale 178, Aprigliano, sul Craticello, affluente del Crati, centro caratterizzato in età bruzia da un toponimo (Argonio) che si riferiva proprio alla posizione nei confronti di questo fiume. Ricco di monumenti, il territorio comunale presenta nella località Ceci un Romitorio costruito nel 1200 da Gioacchino da Fiore su mandato dell'imperatore Federico II, complesso rimasto intatto, affacciato sul Lago Arvo, presso Lorica.

Queste località, che costituiscono veri e propri centri propulsori dello sviluppo del Parco come sistema territoriale, sono i più forti input per una "apertura" dell'area all'esterno e per un potenziamento delle relazioni, in termini organizzativi e socio-culturali, fra lo stesso Parco e i soggetti del territorio in cui questo si inscrive.

Le direttrici lungo le quali si configurano effettive condizioni di "sostenibilità"della crescita locale sono da ricercare essenzialmente in una ottimizzazione delle condizioni di accessibilità, in un ammodernamento e in un riassetto della viabilità che interessa l'area, dalla riqualificazione di sentieri antichi al ridisegno delle arterie più recenti, con l'obiettivo della realizzazione di un effettivo sistema di direttrici che favoriscano gli scambi con i centri urbani e con tutte le strutture insediative "centrali"nel territorio.

È evidente, infatti, come ogni prospettiva credibile di sussistenza e di progressiva affermazione del Parco in quanto sistema che vive nel proprio territorio risieda nella definizione di effettive condizioni di integrazione con le principali città e con l'intera armatura insediativa regionale, in primo luogo con i capoluoghi provinciali. Fra questi Cosenza, a nord-ovest, ha costituito il tradizionale, storico bacino di utenza, in termini sociali e produttivi, dell'altopiano silano, il mercato di affluenza delle risorse rurali e il principale centro di offerta di servizi; a sud, Catanzaro, nel cui territorio è situata attualmente la sede del Parco, ha progressivamente rappresentato la base di riferimento per l'area, in termini di offerta di strutture amministrative e socio-territoriali; fino al consolidamento della città di Crotone come 
soggetto di crescente importanza territoriale perché situato sulla costa ionica, lungo una direttrice a crescente sviluppo turistico-balneare, "ultimo nato"come capoluogo di provincia, a est del Parco, all'estremità della direttrice di traffico che fa capo a Cosenza tagliando il Parco stesso all'altezza delle Riserve Naturali "Tasso-Camigliatello" e "I Giganti della Sila”, fra i Laghi di Ariamacina e Votturino, passando per le località di Fago del Soldato, Moccone, Camigliatello Silano, Croce di Magara e Silvana Mansio, nel cuore della Sila Grande.

In sostanza, i caratteri di sostenibilità specifici in cui l'affermazione e la crescita del Parco, in quanto soggetto di integrazione territoriale, hanno trovato spazio e significato pertinente, trovano fondamento in condizioni di equilibrio di antichi ecosistemi locali e in assetti moderni, e tuttavia inscritti nella storia dei luoghi. Il Parco stesso, infatti, per poter sussistere come unità territoriale organica deve proporsi come sistema di contenuti e input radicati e, come tali, competitivi e strategici, in grado di introdurre il sistema locale in campi relazionali di scala globale. Sostenibilità dei processi che interessano il Parco, dunque, deve significare essenzialmente conoscenza, ovvero educazione e formazione di operatori ed entità locali in grado di diffondere e approfondire gli effettivi contenuti di creatività che le risorse locali propongono, nel quadro di efficaci e operative prospettive di sviluppo dell'intero territorio regionale.

\section{BibLIOGRAFIA}

Agnelli L., Escursione nella Sila, Catanzaro, Tipografia del Pitagora, 1868.

Bevilacqua F., Il Parco Nazionale della Sila. Guida naturalistica ed escursionistica, Soveria Mannelli, Rubbettino Editore, 1999.

Bevilacqua E., Guida Turistica. Sila e dintorni, Cosenza, Dea Editori, 2002.

Bevilacqua F., Quell'angolo di Scandinavia chiamato Sila. Il Parco fantasma diventa realtà, «Calabria», aprile 2003, pp. 28-39.

Bevilacqua F., Calabria, immagini della natura, Catanzaro, Abramo, 1992.

Ciolli M., Parco Nazionale della Calabria, Roma, Reda, 1977.

Donin G., Parchi. Larchitettura del giardino pubblico nel progetto europeo contemporaneo, Cannitello (Reggio Calabria), Biblioteca del cenide, 1999. 
Douglas N., Old Calabria, Londra, Martin Secker, 1915; trad it.: Vecchia Calabria, Firenze, Martello, 1962.

Gambi L., Calabria, Torino, UTET, 1965.

Guida all'uso del parco. Natura, cultura, storia, tradizioni e paesaggi dei Parchi Nazionali d'Italia, Ministero dell'Ambiente e della Tutela del Territorio, Servizio Conservazione della Natura, 2001.

Guido D. (a cura), Le montagne calabresi. Pollino, Catena Costiera, Sila, Regione Calabria, Assessorato alla Forestazione, Soveria Mannelli, Rubbettino Editore, 2002.

Iovino F., La gestione delle pinete di laricio in Sila, inLa gestione forestale nellambito dello sviluppo sostenibile del territorio provinciale (Cupone, Parco Nazionale della Sila, 5 luglio 2003), Cosenza, Unisci, 2003, pp. 27-41.

Istituzione del Parco nazionale della Sila e dell'Ente parco. Decreto del Presidente della Repubblica, 14 novembre 2002, «Gazzetta Ufficiale della Repubblica Italiana», Serie generale-n. 63, 17-3-2003, pp. 4-14.

Luci e ombre della Sila, Amministrazione provinciale di Cosenza, Assessorato al Turismo, Cosenza, Pellegrini Editore, 1998.

Ministero per le Politiche Agricole - Direzione Generale delle Risorse Forestali, Montane ed Idriche, Parco Nazionale della Calabria, Roma, 1968

Mirabelli, P., Guida naturalistica della Calabria, Cosenza, Due Emme, 1989.

Mollica E., Calabria: "area interna", in Viganoni L. (a cura), Temi e problemi di geografia. In memoria di Pietro Mario Mura, Reggio Calabria, Gangemi Editore, 1998, pp. 153-170.

Mura P. M., Calabria. Un'area a "sensibile divario", in Landini P., Salvatori F. (a cura), I sistemi locali delle regioni italiane (1970-1985), in Memorie della Società Geografica Italiana, Roma, vol. XLIII, 1989.

Pratesi F., Tassi F., Guida alla natura di Puglia, Basilicata e Calabria, Milano, Mondadori, 1979.

Scandale G, Una tesi sul Parco della Sila, «Area Locale», Url: http://www. arealocale.com/default.asp? action $=$ article $\& \mathrm{ID}=31$

Strabone, Geografia, VI, 9.

Touring Club Italiano, Guida d'Italia, Basilicata, Calabria, Milano, 1980. 